\title{
Fatores que influenciam o resultado da osteossíntese na fratura do colo do fêmur em pacientes adultos jovens*
}

\section{Factors Influencing the Outcome of Osteosynthesis in the Fracture of the Femoral Neck in Young Adult Patients}

\author{
Daniel Alves Ramallo ${ }^{1}$ Leandro Lemgruber Kropf ${ }^{1}$ Alexandre Dreifus Zaluski ${ }^{1}$ \\ Amanda dos Santos Cavalcanti ${ }^{2}$ Maria Eugenia Leite Duarte ${ }^{2}$ João Antonio Matheus Guimarães ${ }^{1,2(0)}$ \\ ${ }^{1}$ Centro de Trauma, Instituto Nacional de Traumatologia e Ortopedia, \\ Rio de Janeiro, RJ, Brasil \\ ${ }^{2}$ Centro de Pesquisa Clínica, Instituto Nacional de Traumatologia e \\ Endereço para correspondência João Antonio Matheus Guimarães, \\ MD, PhD, Instituto Nacional de Traumatologia e Ortopedia Jamil \\ Ortopedia, Rio de Janeiro, RJ, Brasil \\ Haddad, Av. Brasil, 500, Caju, Rio de Janeiro, RJ, 20940-070, Brasil \\ (e-mail: jmatheusguimaraes@gmail.com).
}

Rev Bras Ortop 2019;54:408-415.

\section{Resumo \\ Palavras-chave \\ - colo do fêmur \\ - necrose da cabeça do fêmur \\ - fraturas do colo femoral \\ - pseudoartrose}

\section{Abstract}

Objetivos Avaliar os fatores que influenciam o resultado da osteossíntese pela redução fechada da fratura do colo femoral nos pacientes jovens.

Métodos Foi feito um estudo retrospectivo com revisão dos dados dos pacientes operados em um hospital ortopédico de grande porte, de 2003 a 2011, com um total de 81 pacientes que atenderam aos critérios de inclusão. $O$ intervalo de tempo entre a fratura e a cirurgia, o desvio inicial da fratura, a qualidade da redução e o posicionamento dos implantes foram os fatores avaliados.

Resultados $\mathrm{O}$ estudo encontrou forte relação entre a qualidade da redução e o sucesso terapêutico. O grau de desvio inicial e o tempo entre o trauma inicial e a osteossíntese não influenciaram o desfecho cirúrgico em relação à consolidação óssea. O correto posicionamento dos implantes mostrou relação com a evolução satisfatória no pós-operatório dos pacientes.

Conclusão A qualidade da redução e o posicionamento dos implantes são fatores que influenciam o resultado da osteossíntese na fratura do colo do fêmur no paciente adulto jovem.

Objectives To evaluate the factors that influence the outcome of osteosynthesis after closed reduction of the fracture of the femoral neck in young adult patients.

Methods A retrospective study was conducted, reviewing the data of patients operated in a large orthopedic hospital from 2003 to 2011; a total of 81 patients met the inclusion criteria. The time interval between the fracture and the surgery, the initial fracture deviation, the quality of the reduction, and the placement of the implant were evaluated.

\footnotetext{
Trabalho desenvolvido no Centro de Atenção Especializada de Trauma (CAE Trauma), Instituto Nacional de Traumatologia e Ortopedia (Into), Rio de Janeiro, RJ, Brasil. Publicado originalmente por Elsevier Editora Ltda.

(1) João Antonio Matheus Guimarães's ORCID is https://orcid.org/00000002-5647-2248.
}

recebido

28 de Setembro de 2017

aceito

22 de Dezembro de 2017
DOI https://doi.org/

10.1016/j.rbo.2017.12.021. ISSN 0102-3616.
Copyright $\odot 2019$ by Sociedade Brasileira License terms de Ortopedia e Traumatologia. Published by Thieme Revnter Publicações Ltda, Rio de Janeiro, Brazil 


\section{Keywords}

- femoral neck

- femoral head necrosis

- femoral neck fractures

- pseudoarthrosis
Results The present study observed a strong relationship between the quality of the reduction and therapeutic success. The degree of the initial deviation and the time elapsed between the initial trauma and the osteosynthesis did not influence the surgical outcome regarding bone consolidation. The correct positioning of the implants was associated with a satisfactory evolution in the postoperative period.

Conclusion The quality of the reduction and the positioning of the implants are factors that influence the results of osteosynthesis in fractures of the femoral neck in young adult patients.

\section{Introdução}

As fraturas do fêmur proximal são um problema de saúde pública que têm associação direta com o aumento das taxas de morbidade e de mortalidade. Apenas $50 \%$ dos pacientes com fratura do fêmur proximal são totalmente reabilitados e retornam às atividades anteriormente desempenhadas. ${ }^{1}$ Em todas as faixas etárias, $\sim 50 \%$ das fraturas do fêmur proximal ocorrem na região do colo do fêmur. As fraturas da cabeça do fêmur e das regiões trocantérica e subtrocantérica correspondem à outra metade, o que demonstra a grande prevalência e o impacto social e econômico dessa fratura no espectro das lesões do fêmur proximal. ${ }^{2}$

A fratura do colo do fêmur tem distribuição bimodal. Nos pacientes com $>60$ anos, a fratura está geralmente associada a um trauma de baixa energia e à diminuição da massa óssea do fêmur proximal. No paciente com $<60$ anos, a fratura está relacionada a um trauma de maior energia e a lesões associadas em outros sistemas. $^{3}$

Os principais objetivos do tratamento da fratura do colo do fêmur são a restauração da anatomia da região, a preservação do estoque ósseo e a rápida recuperação funcional do membro. ${ }^{4}$ Quanto ao tipo de tratamento cirúrgico, pode-se optar pela artroplastia total, parcial, ou osteossíntese, baseado no padrão da fratura e nas características do paciente. As opções de osteossíntese variam desde a fixação com parafusos, fixação com parafuso deslizante do quadril associado à placa tubo, ou placa angulada associada a parafuso antirrotatório.

O emprego da osteossíntese no tratamento desse tipo de fratura ainda está em discussão. Um estudo recente mostrou melhores resultados em pacientes tratados com artroplastia, especialmente naqueles $>65$ anos. $^{5} \mathrm{~A}$ fixação estaria indicada para o tratamento das fraturas não desviadas e nos casos em que, apesar do desvio, o paciente tenha idade $<60$ anos. Nessa situação, a preservação do segmento cefálico do fêmur evitaria a necessidade de artroplastia e suas complicações em um indivíduo jovem. Entretanto, a incidência de complicações da osteossíntese do colo do fêmur, como necrose avascular da cabeça do fêmur (NACF), ausência de consolidação da fratura, e a falha da fixação, tornou esse tema controverso na literatura. ${ }^{6}$ Outra complicação frequente é o encurtamento femoral decorrente da impacção do foco de fratura, que pode determinar alteração no offset do quadril. Zielinski et $\mathrm{al}^{7}$ fizeram um estudo randomizado, multicêntrico, com 76 pacientes submetidos à osteossíntese de fratura do colo do fêmur que apresentavam algum grau de encurtamento após a consolidação da fratura. Eles estudaram o padrão da marcha e a força motora do quadril e compararam com o lado contralateral, concluindo que os pacientes jovens são capazes de compensar o encurtamento resultante da consolidação da fratura do colo femoral e que poucos casos necessitaram do uso de compensação por palmilha.

Um fator apontado como relacionado ao mau prognóstico no tratamento dessa lesão por meio da osteossíntese é o intervalo de tempo elevado entre o momento da fratura e a cirurgia. Acredita-se que quanto mais precocemente a fratura for fixada, melhor será o resultado funcional e menor será a tendência à falha da síntese e à NACF. 0 prognóstico favorável da fixação precoce estaria associado ao rápido reestabelecimento do fluxo sanguíneo local e à redução dos danos secundários. Considerase como tratamento ideal a redução e a fixação da fratura com até 12 horas de evolução. A estabilização cirúrgica com tempo $>24$ horas estaria associada a piores resultados e a uma maior incidência de falha da técnica operatória que, nesse caso, seria atribuída à fixação tardia da lesão. ${ }^{8}$ Outros fatores, como o desvio inicial da fratura, a qualidade da redução obtida durante o tratamento cirúrgico, e o tipo de implante usado, parecem também influenciar diretamente o resultado final do tratamento cirúrgico. ${ }^{9}$ Dessa maneira, informações que auxiliem os cirurgiões na conduta terapêutica dessa fratura são fundamentais para a redução das taxas de complicações e de falha. No presente estudo, identificamos na amostra de pacientes fatores que podem influenciar diretamente no resultado da osteossíntese do colo do fêmur e sugerimos critérios para subsidiar o cirurgião na tomada de decisão sobre o melhor tratamento a ser adotado em tais fraturas, particularmente em adultos jovens.

\section{Material e Métodos}

Entre agosto de 2003 e agosto de 2011, um total de 845 pacientes foram internados em um hospital ortopédico de grande porte para tratamento de fratura do colo do fêmur. Foram incluídos no estudo homens e mulheres $\leq 60$ anos, portadores de fratura do colo femoral relacionada com trauma de alta ou moderada energia, tratados por redução fechada e fixação interna com 3 parafusos canulados, dispostos de acordo com a configuração do triângulo invertido. ${ }^{10}$ Foram excluídos do estudo os pacientes com $<15$ anos, portadores de fratura patológica do colo do fêmur, com artrite séptica, ausência de documentação radiográfica em pelo menos 2 incidências (anteroposterior [AP] e perfil), documentação 
radiográfica de baixa qualidade técnica, diagnóstico NACF anterior à fixação da fratura, tempo entre a fratura e o tratamento cirúrgico $>42$ dias, perda do acompanhamento clínico antes do fim do período de observação de 36 meses, pacientes submetidos a outras técnicas de osteossíntese diferentes da usada no presente estudo ou submetidos à artroplastia do quadril.

Após aplicação dos critérios de inclusão e de exclusão, 81 pacientes constituíram a amostra final, dos 845 selecionados inicialmente. Foram excluídos do estudo 632 (74,8\%) por terem sido submetidos a artroplastia; 112 (13,25\%) foram excluídos por terem sido submetidos a outras técnicas de osteossíntese, e 20 (2,4\%) não tinham documentação médica completa, não cumpriram a rotina de reavaliação ambulatorial, ou estavam fora da faixa etária do estudo.

Trata-se de um estudo observacional, longitudinal, retrospectivo, analítico e comparativo. O estudo foi feito pela revisão dos registros médicos com análise evolutiva dos exames complementares. As lesões foram analisadas em imagens radiográficas com boa qualidade técnica por três observadores independentes com experiência no tratamento de fraturas do fêmur que atuam no Centro de Atenção Especializada de Trauma (CAE Trauma) do hospital. As lesões foram classificadas quanto à presença de desvio inicial por meio da visualização seriada de radiografias simples do quadril, nas incidências em AP e em perfil. Foram estratificadas, segundo os critérios da classificação de Garden, ${ }^{11}$ nos tipos I e II no caso das lesões sem desvio, e nos tipos III e IV nas lesões com desvio.

Todos os pacientes foram submetidos ao mesmo procedimento cirúrgico feito pelo grupo de cirurgiões integrantes do CAE Trauma. A cirurgia foi feita pela redução fechada da fratura, em mesa de tração ortopédica, com controle radioscópico, seguida da fixação interna com 3 parafusos canulados de 7,0 mm. Em nenhum paciente foi feita drenagem do hematoma intracapsular. No pós-operatório, a mobilização do membro acometido era estimulada precocemente, enquanto que a carga parcial era estimulada assim que o paciente a tolerasse. A carga total só era permitida após a confirmação radiográfica da consolidação da fratura.

A qualidade da redução foi avaliada nas duas incidências radiográficas (AP e perfil), e foi considerada satisfatória quando existia um desvio $<2 \mathrm{~mm}$ no foco de fratura e os ângulos de Garden eram de $160^{\circ}$ e $180^{\circ}$, respectivamente. O posicionamento dos parafusos foi considerado adequado quando a distância entre a sua ponta e o osso subcondral era $<5 \mathrm{~mm}$, o parafuso distal estava no nível ou acima do pequeno trocanter, e a angulação entre os parafusos paralelos era $<10^{\circ} .^{12}$

A partir da busca ativa nos prontuários, foram obtidas as informações sobre as características dos pacientes, sobre a lesão (tempo decorrido entre a ocorrência da fratura e o tratamento, o mecanismo do trauma e o grau de desvio inicial da lesão), sobre o tratamento e sobre a evolução clínica do paciente (consolidação, diagnóstico de NACF ou de ausência de consolidação associado à falha da síntese).

Todos os pacientes foram acompanhados por meio de consultas ambulatoriais periódicas por, no mínimo, 36 meses. A rotina de reavaliação seguiu o protocolo do CAE Trauma, que consiste em consultas seriadas com 15 dias, 1 mês, 3 meses, 6 meses, e uma revisão anual; tinha-se como referência a data da cirurgia. O tempo decorrido entre o tratamento e a ocorrência de complicação (ausência de consolidação e NACF) foi obtido para todos os pacientes e expresso em meses.

Quanto à ocorrência de consolidação da fratura, todos os pacientes foram avaliados por exame clínico e por meio da análise de imagens radiográficas nas projeções AP e de perfil do fêmur proximal. Essa avaliação foi feita por três cirurgiões do CAE Trauma, que revisaram, de forma independente, toda a documentação radiográfica, separada em envelopes sem identificação. A consolidação foi definida pela ausência de sintomas no exame clínico e pelo reestabelecimento funcional do paciente, associado à imagem radiográfica de formação óssea, com o desaparecimento da linha de fratura no local da lesão no colo do fêmur. ${ }^{13}$

O diagnóstico clínico de ausência de consolidação foi estabelecido nos pacientes com quadro clínico caracterizado por dor progressiva e incapacidade funcional do membro operado. $O$ diagnóstico radiográfico de ausência de consolidação foi feito por meio da observação da permanência de linha de fratura no colo femoral associada a sinais de falha do implante e de perda da redução.

A confirmação do diagnóstico de NACF foi baseada na classificação de Ficat et al ${ }^{14} \mathrm{e}$ em radiografias convencionais em projeção AP e de perfil. O diagnóstico clínico de NACF foi confirmado nos pacientes com dor progressiva na região inguinal, agravada com esforços físicos.

Após avaliação seriada nos 36 meses subsequentes à cirurgia, os pacientes com fratura consolidada que retornaram às atividades diárias foram alocados no grupo Consolidação. Os pacientes que tiveram necessidade de revisão cirúrgica, fosse por NACF ou por ausência de consolidação, constituíram o grupo Falha.

O Comitê de Ética em Pesquisa do Into aprovou os termos do estudo, que foi isentado da necessidade de assinatura do Termo de Consentimento Livre e Esclarecido.

Os dados numéricos (variáveis quantitativas) foram expressos sob a forma de média \pm desvio padrão (DP), mediana, mínimo e máximo. Os dados categóricos (variáveis qualitativas) foram expressos sob a forma de frequência $(n)$ e percentual (\%). A análise de distribuição dos dados numéricos (distribuição gaussiana ou normal) foi feita pelo teste de Shapiro-Wilk. A comparação entre os grupos de tratamento para as variáveis idade e tempo entre a fratura e o tratamento cirúrgico foi feita pelo teste de Mann-Whitney, pois apresentaram distribuição não normal. A comparação do tempo entre a cirurgia e a detecção da falha foi feita pelo teste-t de Student. Para as análises dos dados categóricos, foi usado o teste exato de Fisher. $O$ risco relativo analisou o risco de desenvolvimento de falha de acordo com os parâmetros referenciais. A análise estatística foi feita no programa GraphPad Prism versão 5.00 para Windows (GraphPad Software, San Diego, CA, EUA).

O preente projeto foi autorizado pelo Comitê de Ética em Pesquisa da nossa instituição e aprovado sob o número 919.66.

\section{Resultados}

Os 81 pacientes foram alocados em dois grupos: Consolidação ou Falha, sendo este último subdividido em NACF ou ausência 
de consolidação, de acordo com a razão do insucesso terapêutico. Dessa forma, 67 pacientes foram incluídos no grupo Consolidação e 14 no grupo Falha (-Fig. 1).

A mediana de idade do grupo Consolidação foi de 44 anos (de 16 a 60 anos) e a do grupo Falha foi de 45 anos (de 19 a 59 anos), sem diferença estatística ( $p=0,955)$.

Os dois grupos foram homogêneos em relação ao gênero, à presença de diabetes mellitus, ao tabagismo e ao risco cirúrgico segundo a Sociedade Americana de Anestesiologia (ASA, na sigla em inglês.). Essas variáveis não influenciaram o desfecho operatório nos grupos estudados (-Tabela $\mathbf{1}$ ).

\section{Relação do Tempo Decorrido Entre a Fratura e o Tratamento Cirúrgico}

A mediana do tempo entre a fratura e a cirurgia no grupo Consolidação foi de 17 dias (de 3 a 40 dias). Nos pacientes do grupo Falha, a mediana foi de 17 dias (de 6 a 42 dias). Não foi observada diferença estatisticamente significativa entre os

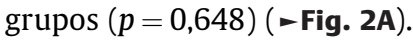

Não foi observada diferença no tempo de internação entre os grupos. A mediana do grupo Falha foi de 6 dias (de 1 a 31 dias). $O$ grupo Consolidação apresentou mediana do tempo de internação de 6 dias (de 2 a 41 dias) (-Fig. 2B).

\section{Influência do Desvio Inicial da Fratura no Desfecho}

Apenas 3 pacientes (3,7\%) não apresentaram desvio da fratura. Observou-se que a totalidade (100\%; 14/14) dos pacientes do grupo Falha apresentou desvio inicial da fratura, e que no grupo Consolidação esse percentual foi de $95,5 \%$ (64/67), e não existiu diferença estatística $(p=1,000)$ (-Fig. 3).

\section{Qualidade da Redução da Fratura}

Em relação à qualidade da redução da fratura, todos os pacientes que evoluíram com consolidação óssea tiveram redução satisfatória (67/67). No grupo que evoluiu para falha terapêutica, 5 pacientes (35,7\%) tiveram redução satisfatória, e 9 (64,3\%) pacientes tiveram redução insatisfatória. 0 risco de falha foi 8,37 vezes maior quando a redução foi inadequada ( $p<0,0001$ ) ( - Fig. 4 ).

\section{Posicionamento dos Implantes}

No grupo Consolidação óssea, 95,5\% (64/67) dos pacientes apresentaram posicionamento adequado dos implantes, o que contrastou com o grupo Falha terapêutica, no qual ocorreu posicionamento inadequado em 85,7\% (12/14) dos casos $(p<0,001)$. O posicionamento correto foi fator de proteção para a ocorrência de falha, evidenciado pelo risco relativo de 0,04 (índice de confiança [IC]: 0,01-0,15) (-Fig. 5).

No grupo Falha, nos seis casos em que ocorreram NACF, a fratura foi classificada como desviada. Entre esses, em dois pacientes, a redução foi satisfatória, com posicionamento adequado dos implantes. Nos demais quatro pacientes, a redução foi insatisfatória e o posicionamento dos implantes foi inadequado.

\section{Tempo Entre o Tratamento da Fratura e a Ocorrência de Complicação}

O tempo entre o tratamento cirúrgico e a detecção da falha terapêutica variou de 5,7 a 49,1 meses. Na análise separada dos desfechos, notou-se que o tempo foi maior para os pacientes que apresentaram NACF $(24,4 \pm 11,4)$ do que para aqueles com ausência de consolidação óssea $(11,3 \pm 4,2)$ (-Fig. 6). Todos os

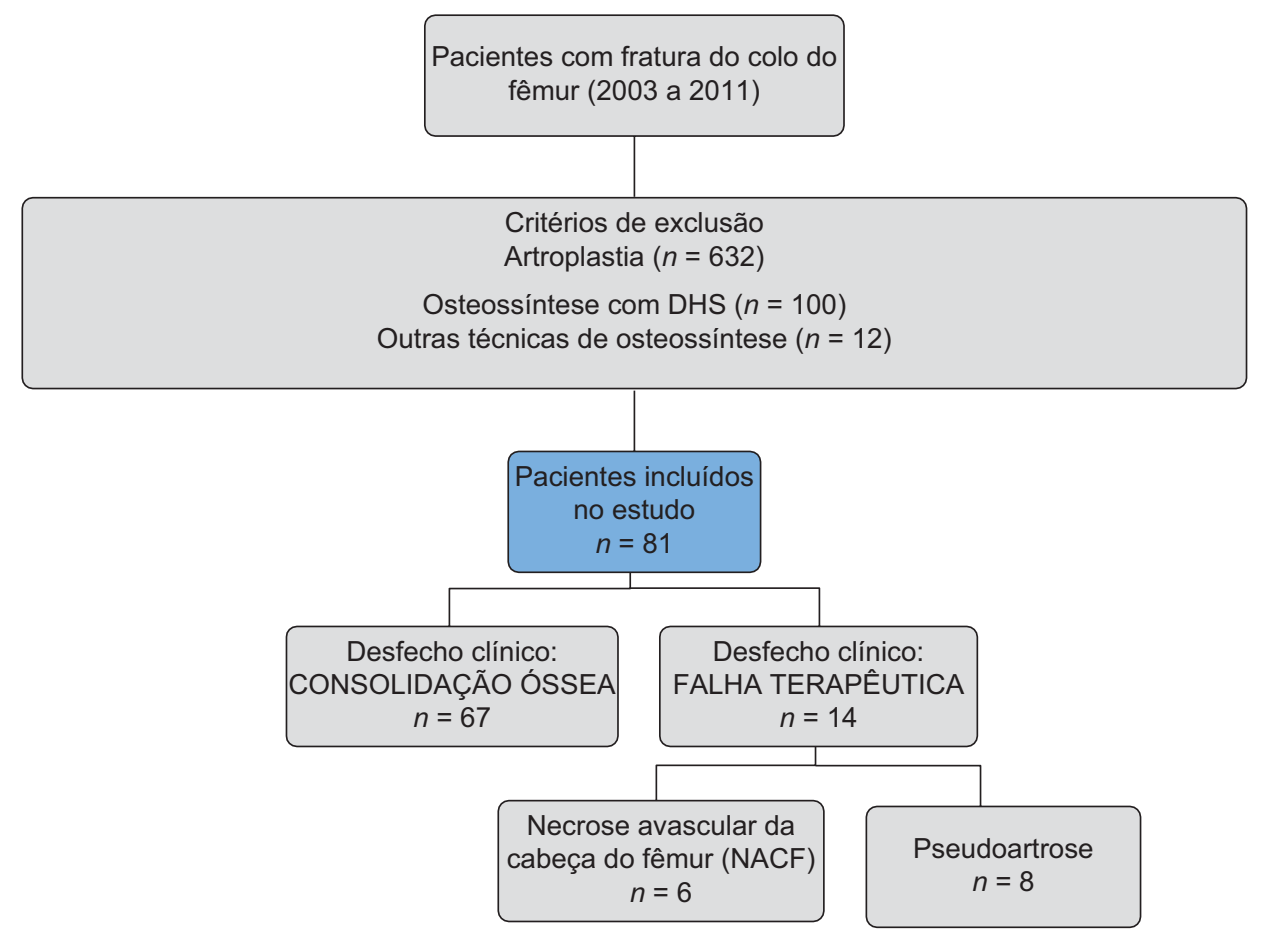

Fig. 1 Desenho experimental, amostra final e grupos de acordo com o desfecho do tratamento. DHS, dynamic hip screw (parafuso deslizante com placa tubo). 
412 Fatores que influenciam o resultado da osteossíntese na fratura do colo do fêmur Ramallo et al.

Tabela 1 Variáveis demográficas categóricas dos pacientes segundo o grupo de desfecho clínico da fratura

\begin{tabular}{|c|c|c|c|c|c|c|}
\hline \multirow[t]{2}{*}{ Variável } & \multirow[t]{2}{*}{ Categoria } & \multicolumn{2}{|c|}{$\begin{array}{l}\text { Consolidação } \\
(n=67)\end{array}$} & \multicolumn{2}{|c|}{ Falha $(n=14)$} & \multirow[t]{2}{*}{ valor-p $p^{a}$} \\
\hline & & $n$ & $\%$ & $n$ & $\%$ & \\
\hline \multirow[t]{2}{*}{ Gênero } & Masculino & & & 10 & 71,4 & 1,000 \\
\hline & Feminino & 22 & 32,8 & 4 & 28,6 & \\
\hline \multirow[t]{2}{*}{ Diabetes mellitus } & Sim & 4 & 6,0 & 0 & 0 & 1,000 \\
\hline & Não & 63 & 94,0 & 14 & 100 & \\
\hline \multirow[t]{2}{*}{$\mathrm{ASA}^{\mathrm{b}}$} & 1 & 28 & 53,8 & 6 & 50,0 & 1,000 \\
\hline & II e III & 24 & 46,1 & 6 & 50,0 & \\
\hline \multirow[t]{2}{*}{ Tabagismo } & Sim & 16 & 23,9 & 5 & 35,7 & 0,502 \\
\hline & Não & 51 & 76,1 & 9 & 64,3 & \\
\hline
\end{tabular}

Abreviação: ASA, escore de risco da Sociedade Americana de Anestesiologia.

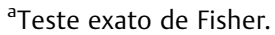

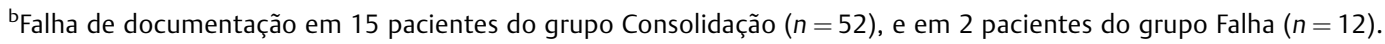
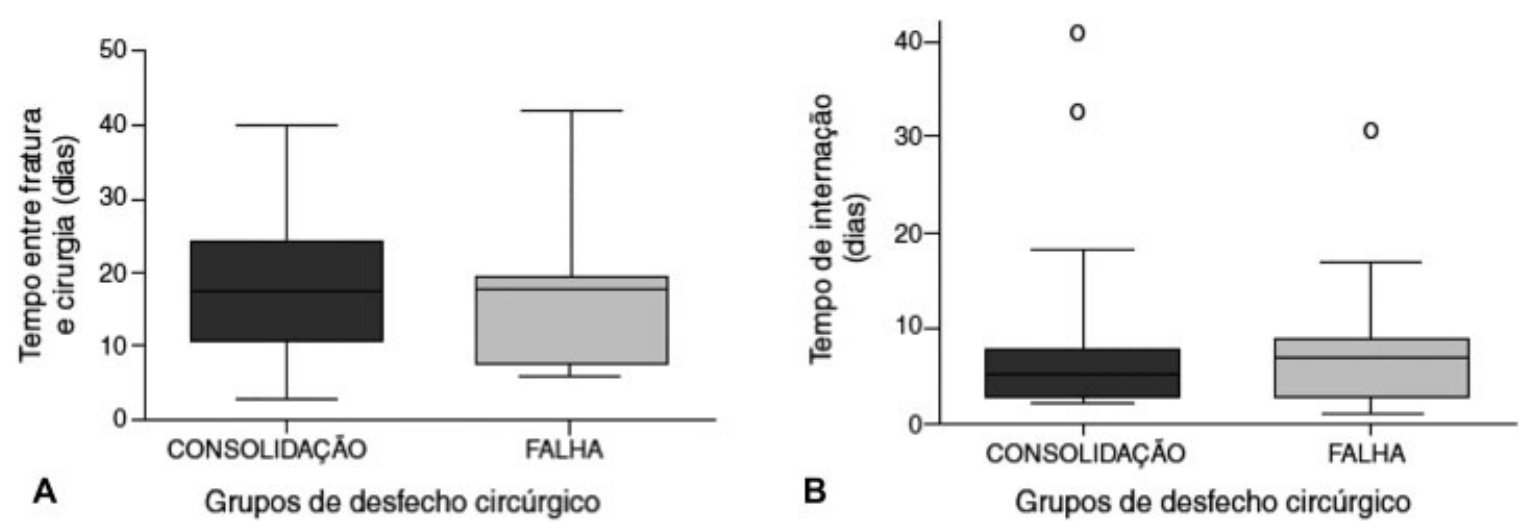

Fig. 2 Acompanhamento dos pacientes com fratura do colo femoral tratados cirurgicamente e que evoluíram com falha terapêutica (necrose avascular da cabeça do fêmur ou ausência de consolidação) ou com consolidação óssea. A, tempo entre a fratura e a cirurgia. B, tempo de internação. As linhas horizontais das extremidades dos retângulos representam os valores da amplitude interquatílica e os extremos das barras representam os valores mínimos e máximos encontrados em cada grupo.

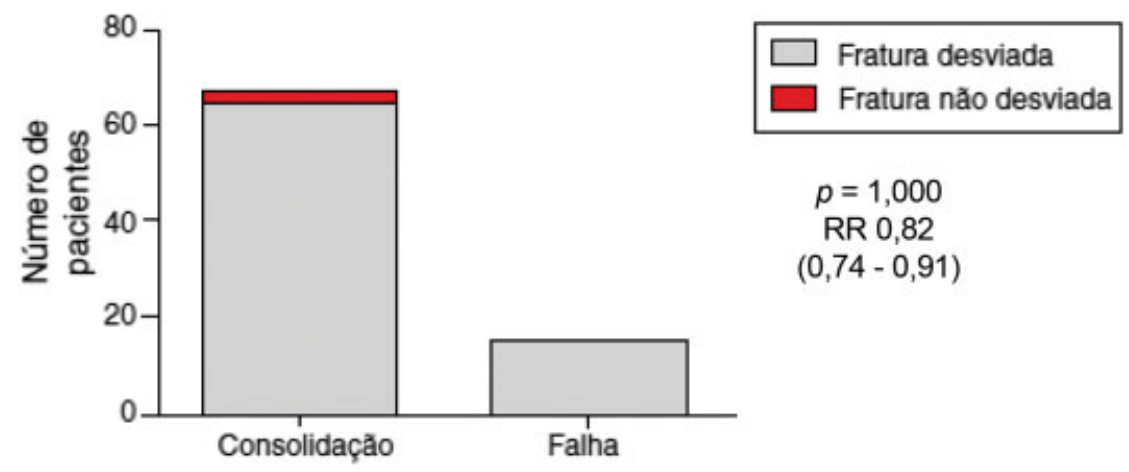

Fig. 3 Influência do desvio da fratura do colo do fêmur no desfecho do tratamento cirúrgico em pacientes jovens. Teste exato de Fisher. RR, risco relativo (intervalo de confiança).

seis casos de NACF foram classificados como estágio III de Ficat et al, ${ }^{14}$ com perda da esfericidade da cabeça femoral, sem lesão acetabular (-Fig. 7).

Todos os pacientes com ausência de consolidação apresentavam fratura desviada. Destes, a redução foi satisfatória em três pacientes, e insatisfatória em cinco. Quanto ao posicionamento dos implantes, ele foi considerado inadequado em todos os casos (-Fig. 8).

\section{Discussão}

A preservação da cabeça femoral pela osteossíntese é desejável no paciente jovem com fratura do colo do fêmur, uma vez que quando ocorre a consolidação óssea sem a presença de NACF, os resultados são satisfatórios e preservam-se a anatomia e a função da articulação. ${ }^{15}$ Entretanto, devido à instabilidade da fratura, associada à dificuldade de se obter uma montagem 


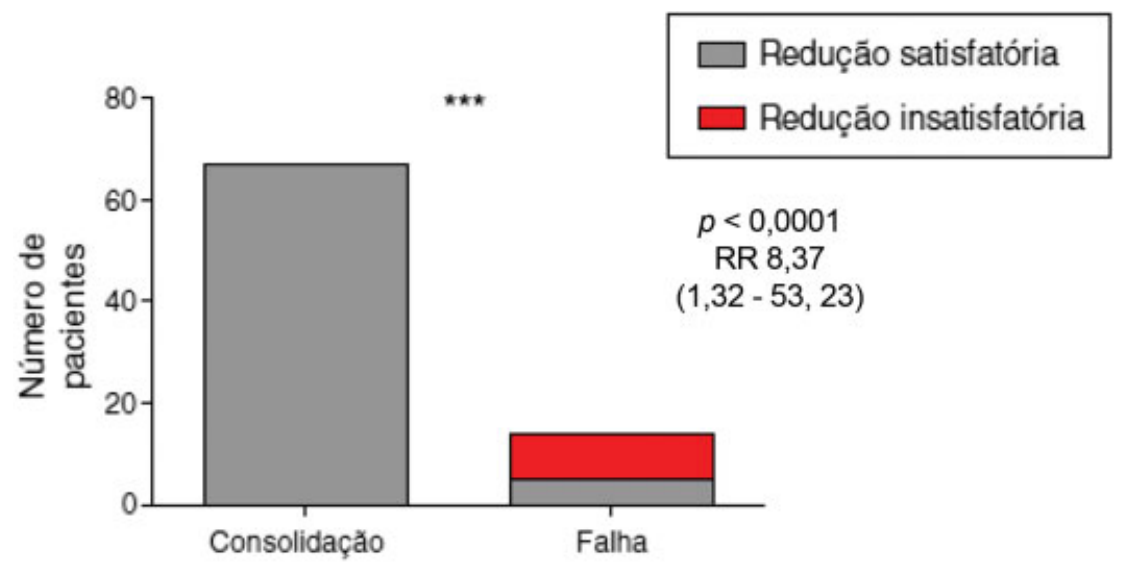

Fig. 4 Desfecho do tratamento cirúrgico da fratura do colo do fêmur em pacientes jovens em função da redução da fratura. A redução foi considerada satisfatória quando o alinhamento foi de $160^{\circ}$ na visão anteroposterior e de $180^{\circ}$ na visão em perfil. Teste exato de Fisher. RR, risco relativo (intervalo de confiança).

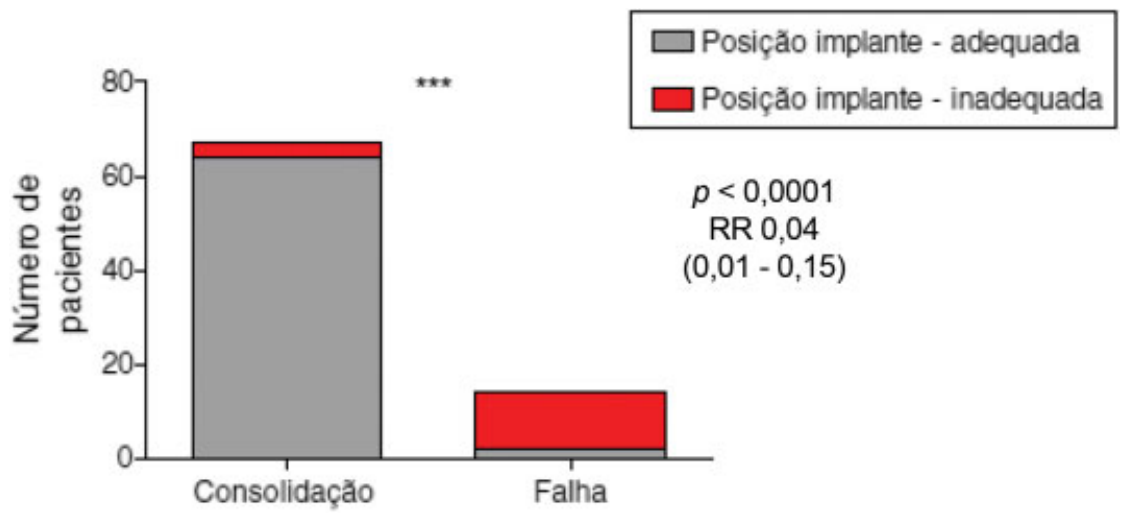

Fig. 5 Desfecho do tratamento cirúrgico da fratura do colo do fêmur em pacientes jovens em função do posicionamento do implante. Teste exato de Fisher. RR, risco relativo (intervalo de confiança).

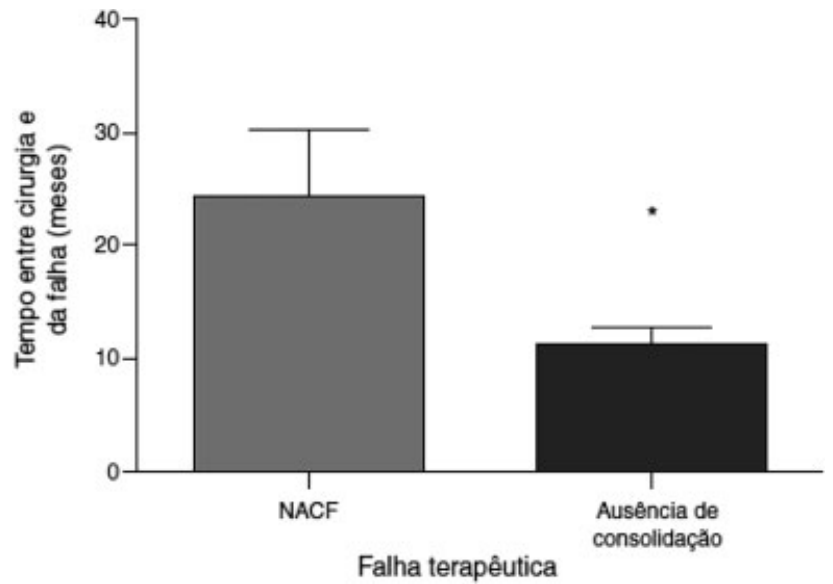

Fig. 6 Tempo entre cirurgia e diagnóstico da falha terapêutica. NACF, necrose avascular da cabeça do fêmur.

estável para a manutenção da redução, a preservação da cabeça femoral pode constituir-se em grande desafio, pela ocorrência de alta incidência de falhas. ${ }^{16} \mathrm{~A}$ redução das taxas de insucesso terapêutico depende de alguns fatores que podem ser controlados, como a qualidade da redução e o posicionamento dos implantes eleitos para a osteossíntese. ${ }^{9}$ Outros fatores são independentes e poderiam influenciar também no resultado final do tratamento, como o grau de desvio inicial da fratura e o tempo decorrido entre a fratura e o tratamento cirúrgico. ${ }^{8}$

O momento ideal para a cirurgia da fratura do colo do fêmur é controverso. Os autores que defendem a cirurgia precoce sugerem que a principal vantagem da redução imediata da fratura é a diminuição da possível compressão dos vasos retinaculares pelo desvio dos fragmentos e a descompressão do hematoma que aumenta a pressão intracapsular, o que melhora o fluxo sanguíneo para a cabeça femoral e minimiza o risco de NACF. ${ }^{8}$ Esses autores revisaram retrospectivamente a fixação precoce de fraturas de colo de fêmur, feita dentro de 12 horas, e a fixação tardia, com mais de 12 horas, em 38 pacientes com idade média de 46 anos. A NACF ocorreu em 16\% dos pacientes submetidos à fixação tardia. De maneira distinta, Upadhyay et al, ${ }^{17}$ em um estudo prospectivo e randomizado com 92 pacientes com $<50$ anos e portadores de fratura do colo do fêmur, não observaram diferença significativa na taxa de consolidação e na incidência de NACF em relação à cirurgia nas primeiras 48 horas após o trauma.

Em nosso trabalho, o tempo decorrido entre a fratura e o tratamento cirúrgico não influenciou o desfecho clínico. Resultados semelhantes foram obtidos em outras séries, ${ }^{9,18}$ as quais também não observaram relação entre fixação precoce e evolução favorável da lesão. 

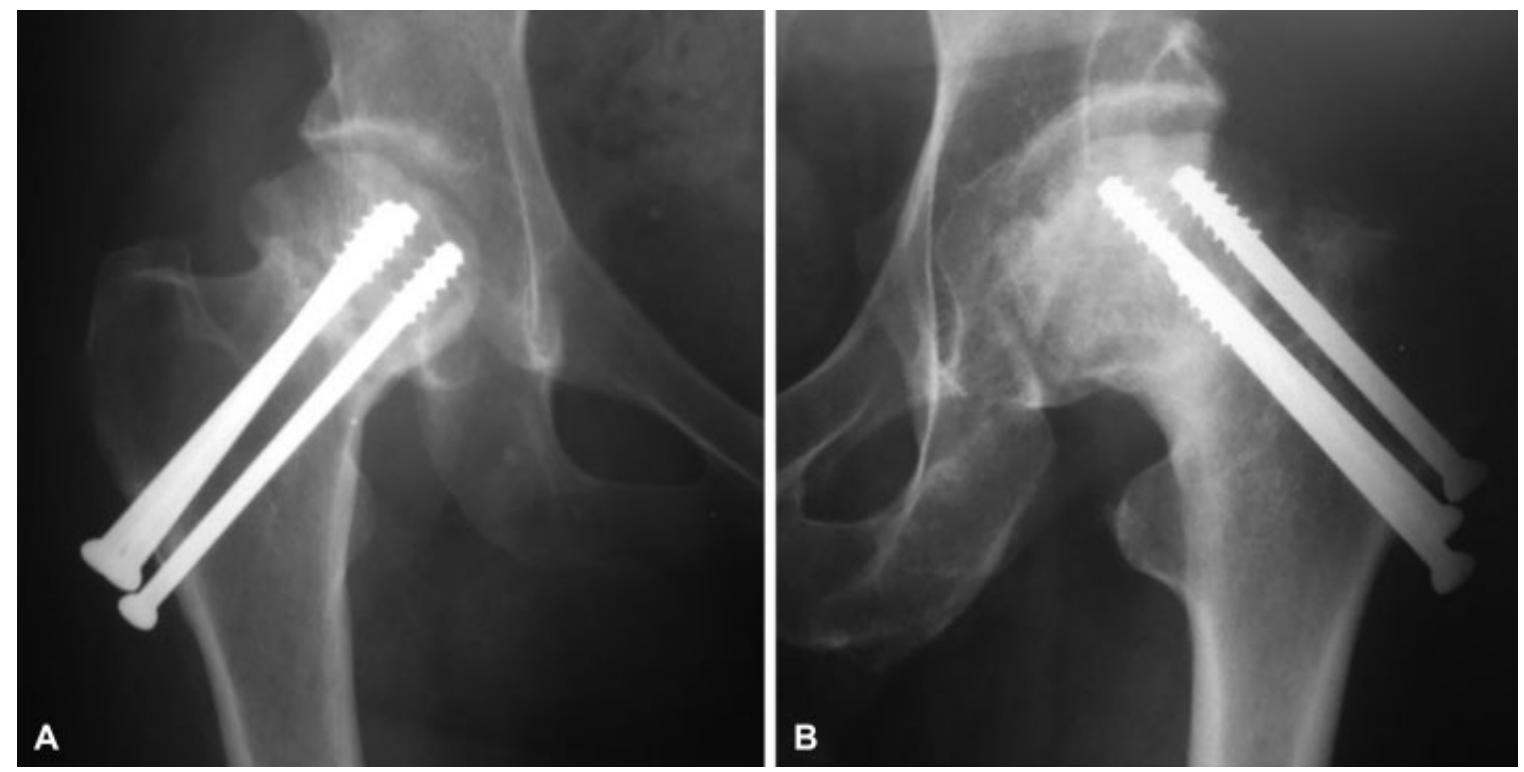

Fig. 7 Necrose avascular da cabeça do fêmur. A, paciente com redução satisfatória e posicionamento adequado dos implantes. B, paciente com redução insatisfatória e posicionamento inadequado dos implantes.
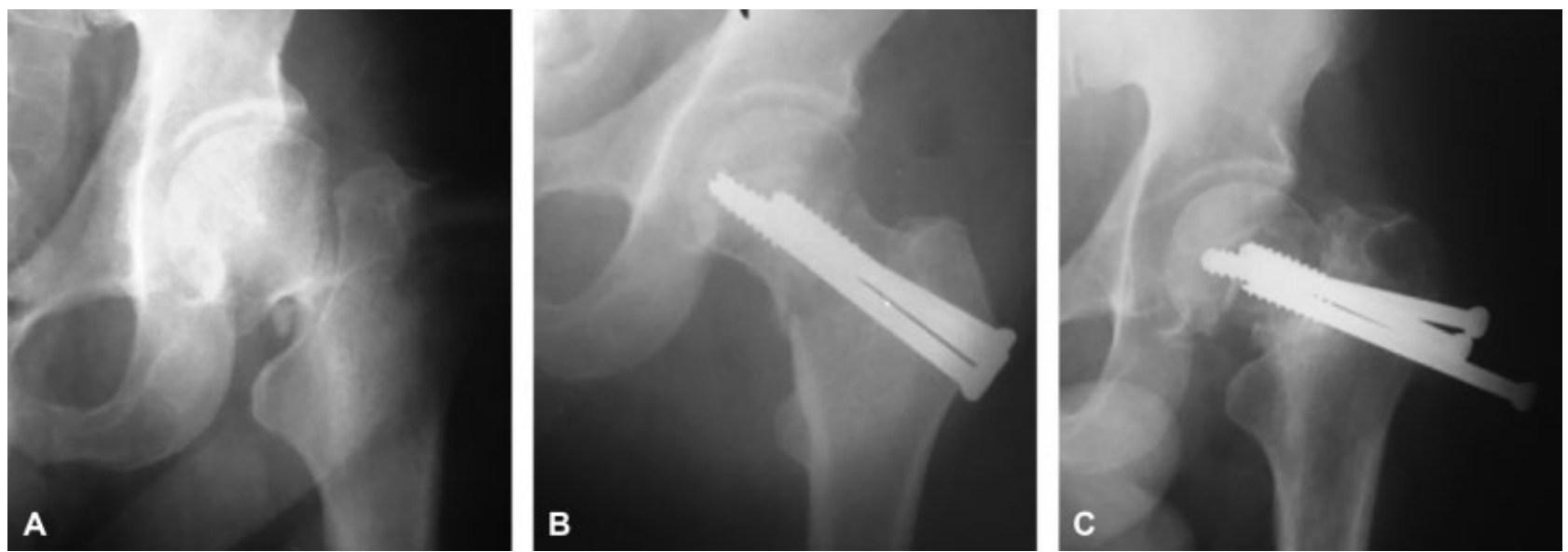

Fig. 8 Evolução radiográfica da fratura do colo femoral em paciente que não apresentou consolidação. A, fratura desviada. B, tratamento cirúrgico com redução satisfatória e posicionamento inadequado dos implantes. C, evolução para ausência de consolidação óssea e falha da osteossíntese.

Em uma revisão feita por Papakostidis et al, ${ }^{19}$ os autores concluíram que a fixação tardia, com mais de 24 horas, tem relação com o aumento na incidência de pseudoartrose, porém não encontraram relação com a evolução para NACF. Nosso estudo não observou maior incidência de pseudoartrose nos pacientes tratados com fixação tardia. Entretanto, nossa amostra foi composta por pacientes com $>3$ dias de evolução da fratura, o que dificulta a comparação com os resultados da referida revisão. ${ }^{19}$

Uma consideração importante a ser discutida é a drenagem do hematoma da fratura. $\mathrm{O}$ relato clássico de Swiontkowski et $\mathrm{al}^{20}$ preconiza a descompressão capsular como prática importante para evitar a necrose avascular da cabeça do fêmur. Esses autores acreditavam que a pressão capsular aumentada levaria à lesão vascular local e à subsequente necrose do tecido ósseo. Porém, Wong et al ${ }^{21}$ mostraram que tal prática deveria ser desencorajada, pois não observaram associação entre a drenagem prévia do hematoma e uma redução nas taxas de necrose. Eles relacionaram a incidência de necrose ao trauma inicial e ao grau de desvio da fratura, responsáveis pela ruptura dos vasos retinaculares no momento da lesão.

Em nenhum paciente do nosso estudo foi feito o procedimento de drenagem do hematoma capsular, as taxas de NACF não foram elevadas e se mantiveram de acordo com a literatura. Kakar et $\mathrm{al}^{22}$ publicaram um estudo multinacional, que corroborou nossa prática, no qual os autores avaliaram a rotina dos cirurgiões no tratamento da fratura do colo do fêmur. Neste estudo, $90 \%$ dos cirurgiões relataram não fazer aspiração do hematoma da fratura previamente ao procedimento de osteossíntese, e enfatizaram que a qualidade da redução e o implante usado seriam os fatores mais importantes para o resultado satisfatório.

Alguns autores acreditam que o desvio inicial da fratura, o qual está associado ao grau de energia do trauma, apresenta relação direta com o desfecho operatório. ${ }^{23-25} \mathrm{Na}$ nossa série de casos, esse fator não pode ser comprovado, pois do total da amostra $(n=81)$, apenas 3 pacientes apresentavam fraturas sem desvio. É possível que, por se tratar de uma amostra 
constituída por pacientes jovens, com melhor qualidade óssea, seria necessário um trauma de alta ou moderada energia para acarretar a fratura desviada. Acreditamos que o desvio inicial possa ter relação com isquemia, e que a NACF seja resultado da relação anatômica intima da região com os vasos retinaculares, ou seja, da alta energia do trauma. Porém, nosso estudo não permite afirmar ou afastar essa correlação.

A redução dentro dos padrões descritos por Garden ${ }^{11}$ parece ser um fator determinante na evolução pós-operatória das fraturas do colo do fêmur submetidas à osteossíntese. Diversos estudos relatam taxas de sucesso superiores quando é obtida uma boa redução. ${ }^{8,26,27}$

Em nosso estudo, encontramos resultados semelhantes, pois a redução satisfatória teve forte associação com a consolidação óssea da fratura. Nos pacientes em quem a redução não foi adequada, a chance de falha foi 8,3 vezes maior do que nos pacientes com redução adequada. Acreditamos que o alinhamento correto da fratura com correção do varo e da retroversão seja primordial no tratamento da fratura do colo do fêmur e possibilite um ambiente mecânico favorável para a consolidação óssea. ${ }^{9}$

\section{Conclusão}

Os fatores mais importantes e que influenciam o resultado final da osteossíntese na fratura de colo do fêmur nos pacientes jovens são a qualidade da redução e a correta aplicação da técnica operatória. O tempo decorrido entre a fratura e a cirurgia não parece ter relação com o desfecho operatório. Nosso estudo não possibilitou afirmar se o desvio inicial foi um fator determinante no desfecho operatório.

Conflitos de Interesses

Os autores declaram não haver conflitos de interesses.

\section{Referências}

1 Loizou CL, Parker MJ. Avascular necrosis after internal fixation of intracapsular hip fractures; a study of the outcome for 1023 patients. Injury 2009;40(11):1143-1146

2 Karagas MR, Lu-Yao GL, Barrett JA, Beach ML, Baron JA. Heterogeneity of hip fracture: age, race, sex, and geographic patterns of femoral neck and trochanteric fractures among the US elderly. Am J Epidemiol 1996;143(07):677-682

3 Protzman RR, Burkhalter WE. Femoral-neck fractures in young adults. J Bone Joint Surg Am 1976;58(05):689-695

4 Stockton DJ, Lefaivre KA, Deakin DE, et al. Incidence, magnitude, and predictors of shortening in young femoral neck fractures. J Orthop Trauma 2015;29(09):e293-e298

5 Rogmark C, Fenstad AM, Leonardsson O, et al. Posterior approach and uncemented stems increases the risk of reoperation after hemiarthroplasties in elderly hip fracture patients. Acta Orthop 2014;85(01):18-25

6 Heetveld MJ, Rogmark C, Frihagen F, Keating J. Internal fixation versus arthroplasty for displaced femoral neck fractures: what is the evidence? J Orthop Trauma 2009;23(06):395-402

7 Zielinski SM, Keijsers NL, Praet SF, et al; FAITH Trial Investigators. Femoral neck shortening after internal fixation of a femoral neck fracture. Orthopedics 2013;36(07):e849-e858

8 Jain R, Koo M, Kreder HJ, Schemitsch EH, Davey JR, Mahomed NN. Comparison of early and delayed fixation of subcapital hip fractures in patients sixty years of age or less. J Bone Joint Surg Am 2002;84(09):1605-1612

9 Araujo TP, Guimarães TM, Andrade-Silva FB, Kojima KE, Silva JdosS. Influence of time to surgery on the incidence of complications in femoral neck fracture treated with cannulated screws. Injury 2014;45(05, Suppl 5):S36-S39

10 Asnis SE, Wanek-Sgaglione L. Intracapsular fractures of the femoral neck. Results of cannulated screw fixation. J Bone Joint Surg Am 1994;76(12):1793-1803

11 Garden RS. The structure and function of the proximal end of the femur. J Bone Joint Surg Br 1961;43(03):576-589

12 Lindequist S, Törnkvist H. Quality of reduction and cortical screw support in femoral neck fractures. An analysis of 72 fractures with a new computerized measuring method. J Orthop Trauma 1995;9 (03):215-221

13 Frank T, Osterhoff G, Sprague S, Garibaldi A, Bhandari M, Slobogean GP; FAITH Investigators. The Radiographic Union Score for Hip (RUSH) Identifies Radiographic Nonunion of Femoral Neck Fractures. Clin Orthop Relat Res 2016;474(06):1396-1404

14 Ficat RP, Arlet J. Forage-biopsie de la tete femorale dans I'osteonecrose primative, Observations histo-pathologiques portant sur huit forages. Rev Rhum 1964;31:257-264

15 Bosch U, Schreiber T, Krettek C. Reduction and fixation of displaced intracapsular fractures of the proximal femur. Clin Orthop Relat Res 2002;(399):59-71

16 Heetveld MJ, Raaymakers EL, Luitse JS, Gouma DJ. Rating of internal fixation and clinical outcome in displaced femoral neck fractures: a prospective multicenter study. Clin Orthop Relat Res 2007;454(454):207-213

17 Upadhyay A, Jain P, Mishra P, Maini L, Gautum VK, Dhaon BK. Delayed internal fixation of fractures of the neck of the femur in young adults. A prospective, randomised study comparing closed and open reduction. J Bone Joint Surg Br 2004;86(07):1035-1040

18 Razik F, Alexopoulos AS, El-Osta B, et al. Time to internal fixation of femoral neck fractures in patients under sixty years-does this matter in the development of osteonecrosis of femoral head? Int Orthop 2012;36(10):2127-2132

19 Papakostidis C, Panagiotopoulos A, Piccioli A, Giannoudis PV. Timing of internal fixation of femoral neck fractures. A systematic review and meta-analysis of the final outcome. Injury 2015;46(03):459-466

20 Bhandari M, Tornetta P III, Hanson B, Swiontkowski MF. Optimal internal fixation for femoral neck fractures: multiple screws or sliding hip screws? J Orthop Trauma 2009;23(06):403-407

21 Wong TC, Yeung SH, Ip FK. The effectiveness of capsular decompression for internal fixation of intracapsular hip fractures. J Orthop Surg (Hong Kong) 2007;15(03):282-285

22 Kakar S, Tornetta P III, Schemitsch EH, et al; International Hip Fracture Research Collaborative. Technical considerations in the operative management of femoral neck fractures in elderly patients: a multinational survey. J Trauma 2007;63(03):641-646

23 Crosby JM, Parker MJ. Femoral neck collapse after internal fixation of an intracapsular hip fracture: Does it indicate a poor outcome? Injury 2016;47(12):2760-2763

24 Kahlenberg CA, Richardson SS, Schairer WW, Cross MB. Rates and Risk Factors of Conversion Hip Arthroplasty After Closed Reduction Percutaneous Hip Pinning for Femoral Neck Fractures-A Population Analysis. J Arthroplasty 2018;33(03):771-776

25 Gardner S, Weaver MJ, Jerabek S, Rodriguez E, Vrahas M, Harris M. Predictors of early failure in young patients with displaced femoral neck fractures. J Orthop 2014;12(02):75-80

26 Haidukewych GJ, Rothwell WS, Jacofsky DJ, Torchia ME, Berry DJ. Operative treatment of femoral neck fractures in patients between the ages of fifteen and fifty years. J Bone Joint Surg Am 2004; 86(08):1711-1716

27 Yang JJ, Lin LC, Chao KH, et al. Risk factors for nonunion in patients with intracapsular femoral neck fractures treated with three cannulated screws placed in either a triangle or an inverted triangle configuration. J Bone Joint Surg Am 2013;95(01):61-69 